\title{
No difference between Hashimoto's thyroiditis and Graves' disease in the association of other autoimmune conditions - systematic review and meta-analysis
}

\author{
Greta Pham-Dobor ${ }^{1}$, Lilla Hanak ${ }^{1}$, Peter Hegyi ${ }^{1}$, Katalin Marta ${ }^{1}$, Andrea Parniczky ${ }^{1}$, \\ Marin Gergics ${ }^{1}$, Patricia Sarlos ${ }^{1}$, Balint Eross ${ }^{1}$, and Emese Mezosi ${ }^{1}$ \\ ${ }^{1}$ University of Pecs Medical School
}

May 5, 2020

\begin{abstract}
Objective: In autoimmune polyglandular syndromes (APS) both types of Autoimmune Thyroid Disorders (AITDs), i.e. Hashimoto's thyroiditis (HT) and Graves' disease (GD) can be present. Design: In this meta-analysis, we aimed to provide the first comprehensive overview of the differences between HT and GD in APS II and III. Methods: Using the MEDLINE and Embase databases all studies containing the keywords of APS II and APS III were screened. Out of 479 studies 18 records containing a total of 1312 patients fulfilled the criteria of our study and were selected for analysis. Meta-analysis was performed using the random-effects model. Results of each meta-analysis were displayed graphically using forest plots. Results: AITDs were detected in $87.8 \%$ of APS patients. HT and GD were specified in 279 and 151 cases, respectively. In the remaining 309 cases, the diagnosis was AITD, without any further characterization. The prevalence of HT, GD and AITD did not differ among APS patients. The pattern of co-associated endocrine, non-endocrine organ-specific and systemic autoimmune disorders was similar in HT and GD. T1DM and AD were found in larger proportion of patients, $70.7 \%$ and $18.5 \%$, respectively. Other autoimmune conditions occurred in $<4 \%$. The majority of autoimmunities occurred in dual combinations $(91.8 \%)$. The combination of four and more autoimmune disorders was published only in HT, in $0.1 \%$ of patients. Conclusions: Using a meta-analysis, no difference could be observed in the prevalence of HT and GD among APS patients and no distinct pattern of co-associated autoimmunities could be established.
\end{abstract}

\section{Hosted file}

manuscript2020.02.27.pdf available at https://authorea.com/users/301155/articles/430962no-difference-between-hashimoto-s-thyroiditis-and-graves-disease-in-the-association-ofother-autoimmune-conditions-systematic-review-and-meta-analysis 


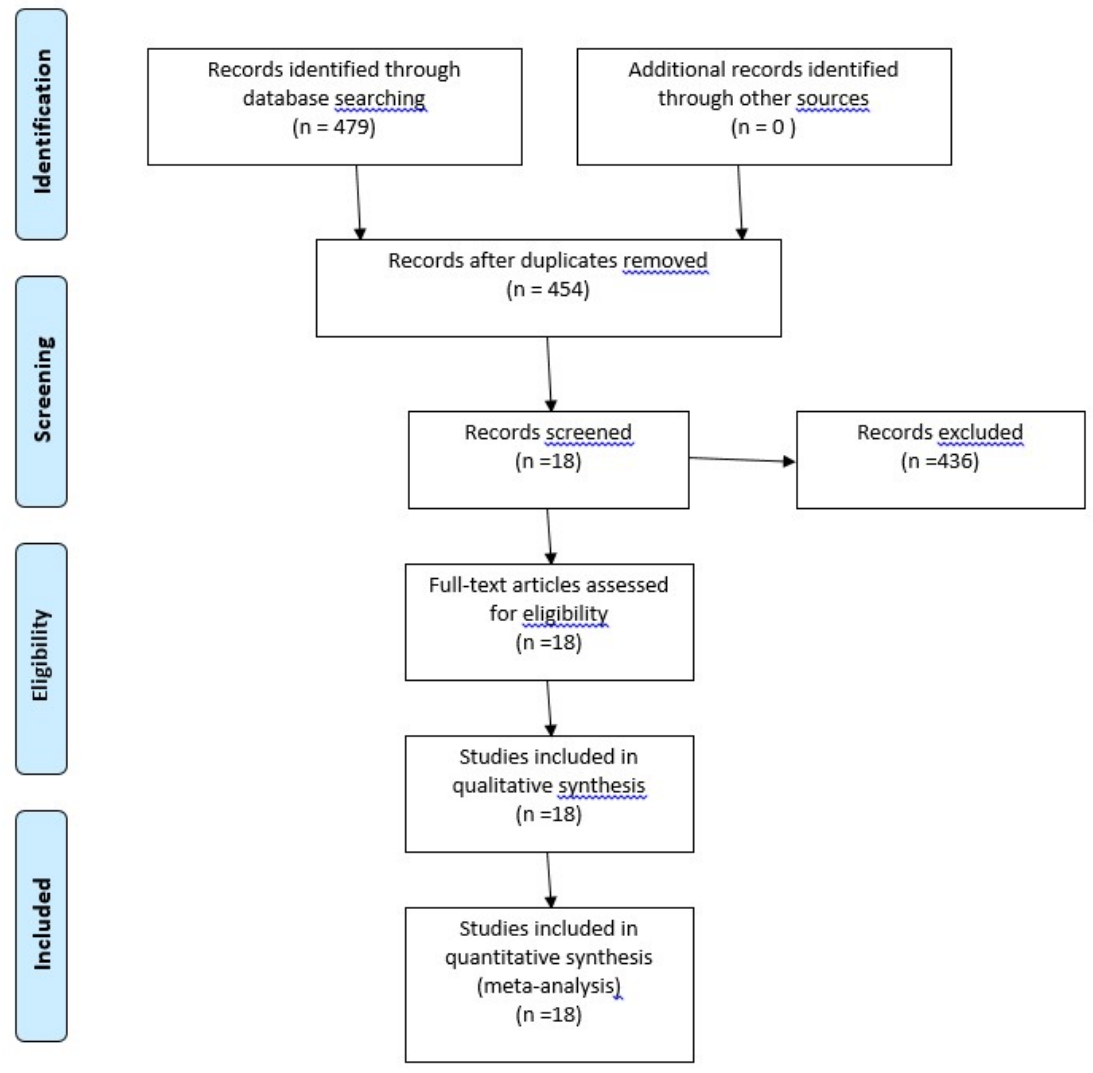




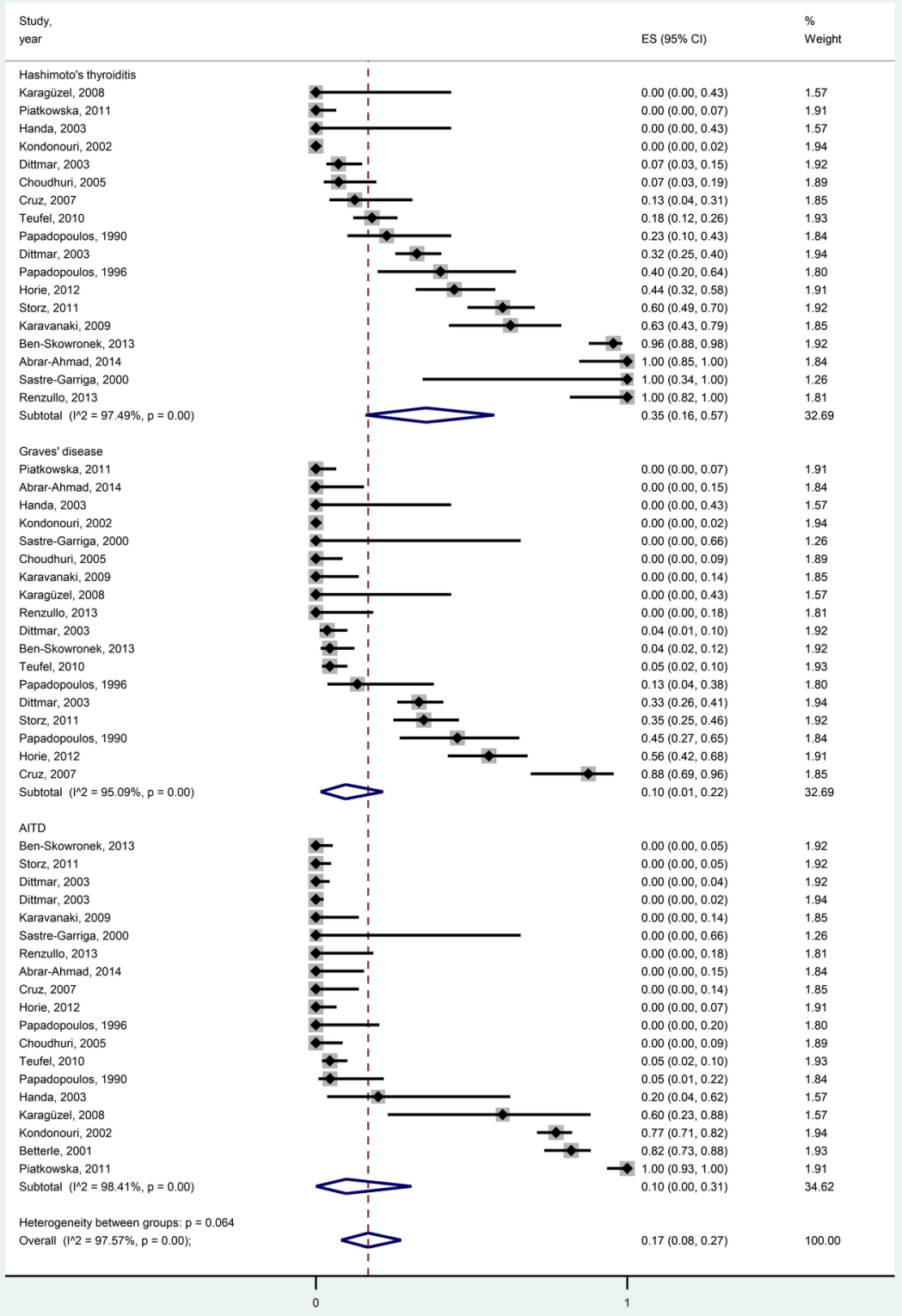




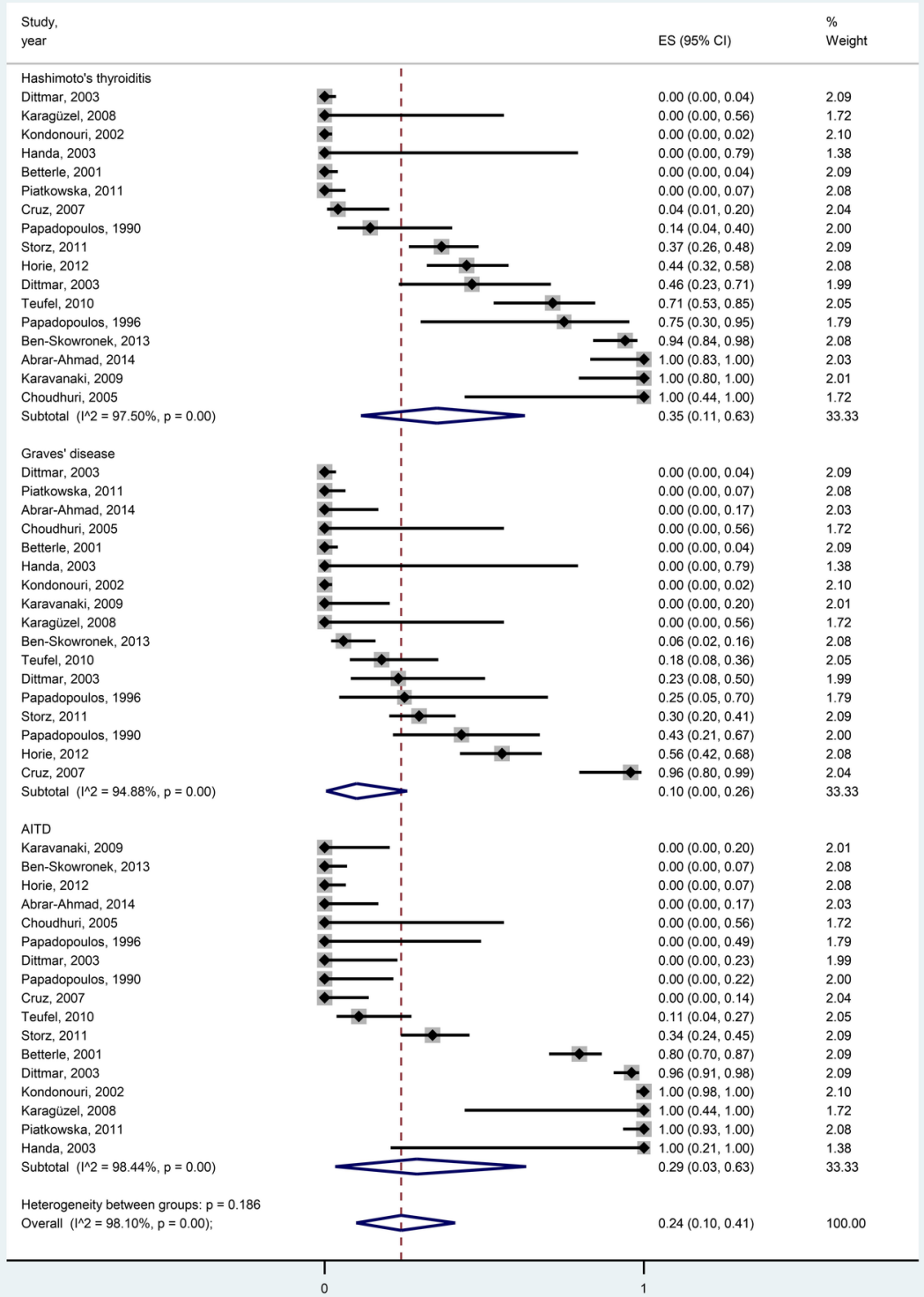




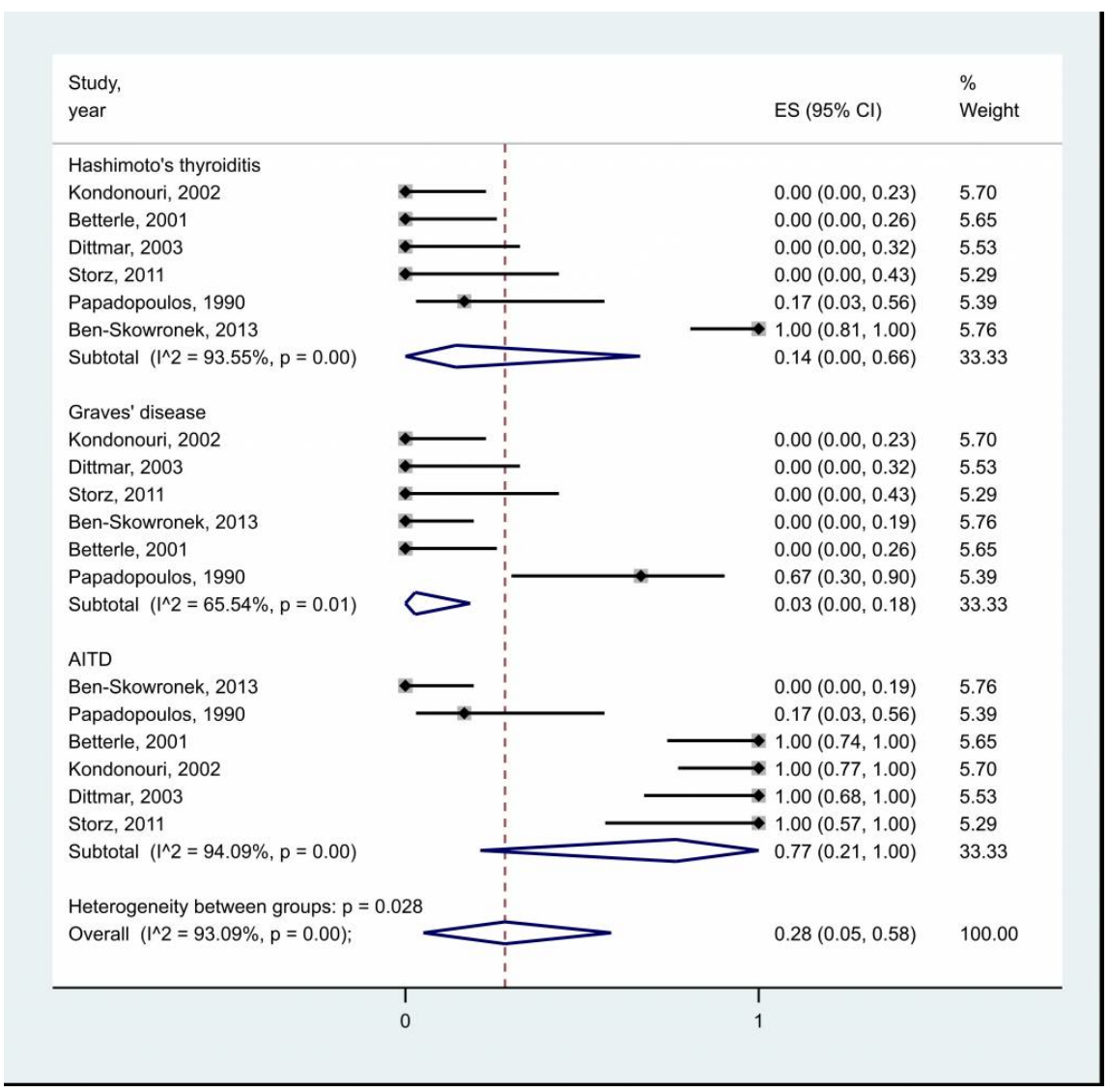

